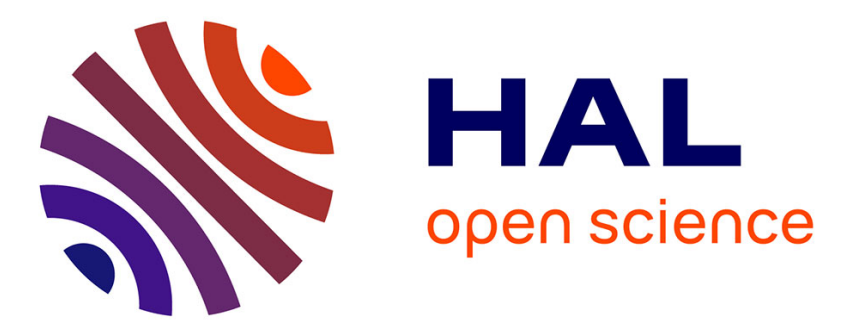

\title{
Shape Optimization for the Design of Passive Mid-Infrared Photonic Components
}

Nicolas Lebbe, Alain Glière, Karim Hassan, Charles Dapogny, Edouard Oudet

\section{To cite this version:}

Nicolas Lebbe, Alain Glière, Karim Hassan, Charles Dapogny, Edouard Oudet. Shape Optimization for the Design of Passive Mid-Infrared Photonic Components. Optical and Quantum Electronics, 2019, 51, pp.166. 10.1007/s11082-019-1849-1 . cea-02095365

\section{HAL Id: cea-02095365 https://hal-cea.archives-ouvertes.fr/cea-02095365}

Submitted on 10 Apr 2019

HAL is a multi-disciplinary open access archive for the deposit and dissemination of scientific research documents, whether they are published or not. The documents may come from teaching and research institutions in France or abroad, or from public or private research centers.
L'archive ouverte pluridisciplinaire HAL, est destinée au dépôt et à la diffusion de documents scientifiques de niveau recherche, publiés ou non, émanant des établissements d'enseignement et de recherche français ou étrangers, des laboratoires publics ou privés. 


\title{
Shape Optimization for the Design of Passive Mid-Infrared Photonic Components
}

\author{
Nicolas Lebbe • Alain Glière • Karim Hassan . \\ Charles Dapogny • Edouard Oudet
}

Received: date / Accepted: date

\begin{abstract}
Shape optimization techniques were first developed in the context of mechanical engineering and, more recently, applied to photonic components for data communication. Here, motivated by the growing application potential of mid-infrared photonics driven by chemical sensing and spectroscopy, we present the design by shape optimization of passive components operating in this wavelength range. A focus is placed on the creation of designs that are fabricable and robust to manufacturing uncertainties.
\end{abstract}

Keywords shape optimization $\cdot$ level-set $\cdot$ mid-IR photonics

\section{Introduction}

Driven by data communication in the near-IR wavelength range, silicon photonics has experienced tremendous developments in the last few decades. The transposition of this scientific and industrial success to the mid-IR (wavelengths comprised between $3 \mu \mathrm{m}$ and $12 \mu \mathrm{m}$ ) has been envisioned some time ago [21] and is currently under rapid progress, with noteworthy developments in materials, passive and active components, laser sources, photodetectors and sensors [6]. Due to the strong absorption bands situated in this wavelength range, applications are found, for instance, in trace gas sensing, spectroscopy and imaging of biological tissues [6].

In the last decades, shape optimization techniques, boosted by additive manufacturing technologies, have been successfully applied to mechanical engineering [2]. More recently, shape optimization appeared in the domain of silicon photonics, first with simulations and designs of passive devices $[8,12]$ and then with micro-fabrication and experimental demonstrations [15, 19,7].

In the context of optimal design of photonic components, most contributions rely on density methods $[8,12]$. This popular paradigm, originally introduced in the context of structural mechanics as a heuristic approximation of mathematical homogenization, and known in this context as the SIMP method [3], amounts to trade the conventional "black-and-white" representation of a design for a "grayscale" density function, with continuous values in the whole interval $[0,1]$. This considerably simplifies the optimization process, but raises the need for an approximate modeling of the physical equations, which have to be expressed in terms of the density function, as well as issues about the interpretation of the resulting design, and notably of its "grayscale" regions, where the density function takes intermediate values in $(0,1)$.

On the other hand, more "geometric" methods, featuring a clear representation of the boundary of the optimized shape, have been considered, relying for instance on the concepts of shape and topological

N. Lebbe

Univ. Grenoble Alpes, CEA, LETI, F38000 Grenoble, France

E-mail: nicolas.lebbe@cea.fr

A. Glière, K. Hassan

Univ. Grenoble Alpes, CEA, LETI, F38000 Grenoble, France

C. Dapogny, E. Oudet

Univ. Grenoble Alpes, CNRS, Grenoble INP, LJK, 38000 Grenoble, France 
derivatives $[10]$ (see also $[17,1]$ for mathematical references): the shape is iteratively modified by either deforming its contour or by inserting small holes inside it. Our present work follows an approach of this kind [11].

To our knowledge, only data communication oriented devices, that is components operating in the near-IR have been investigated so far. The scope of this paper is thus to demonstrate the capabilities of shape derivative-based optimization techniques in the mid-IR, where specific material and technological constraints are present. To begin with, several dielectric materials commonly used in near-IR range are absorption limited in the mid-IR, in particular silica for wavelengths above $4 \mu \mathrm{m}$. This, together with the constraint of full compatibility with CMOS foundries, limits the possible choice to a handful of materials, such as silicon, germanium, silicon-germanium alloys and to a lesser extent chalcogenides [6]. Besides, no single material, except germanium, covers the whole mid-IR wavelength range. Thus, the considerable task of driving to maturity several new photonic platforms, and the associated libraries of components, has to be undertaken. At first glance, wavelengths being larger than in the near-IR, the technological problem seems to escape the race towards smaller critical dimensions and to be simpler to address. However, the design requirements, especially when shape optimization is used, finally end up with similar width to depth aspect ratios and demanding critical dimensions, which must be achieved with materials that are often softer, fragile, water-soluble, etc. Several approaches are currently under investigation regarding passive devices. Each comes along with distinctive features, not found in the near-IR such as, for instance, the realization of complex suspended silicon waveguiding structures [23] or the perfect etching of waveguides in thick and defect free layers of silicon-germanium alloys [5]. In the latter case, getting several micrometers deep vertical sidewalls and low surface roughness requires fine tuning in the choice, thickness and exposure of photoresist, use of dry masks, and precise adjustments of the stripping and chemical mechanical polishing processes.

After a brief description of the model used to solve the direct electromagnetic problem and a summary of our shape derivative-based optimization technique, we present a few passive components designed for operation in the mid-IR. A focus is then placed on the important issues of device fabricability and robustness to manufacturing uncertainties.

\section{Photonic model}

\subsection{The technological platform}

The semiconductor platform used as a basis for our design work is composed of a $1.4 \mu \mathrm{m}$ thick layer of silicon-germanium alloy $\left(\mathrm{Si}_{60} \% \mathrm{Ge}_{40} \%\right.$ ), chosen for its transparency up to the wavelength of $8 \mu \mathrm{m}$, placed on a $2 \mu \mathrm{m}$ thick non stoechiometric silicon nitride $\left(\mathrm{SiN}_{x}\right)$ layer. The waveguides and photonic building blocks are fully etched in the SiGe layer and are surrounded with air (Fig. 1). Based on a reasonably achievable etching aspect ratio of 5, the expected resolution of the fabrication process is better than 300 $\mathrm{nm}$.

As a concrete example of sensing in the mid-IR, we develop thereafter our optimization scheme for a wavelength of $6.06 \mu \mathrm{m}$ in the TM polarization, corresponding to the state of the art of quantum cascade laser as light source for sensing in milk protein detection systems. The values of the optical indices used for the computations are 3.54 for the $\mathrm{Si}_{60} \% \mathrm{Ge}_{40} \%$ alloy and 1.70 for the silicon nitride lower cladding, as measured by ellipsometry in our facilities. In these conditions, $1.4 \mu \mathrm{m} \times 1.4 \mu \mathrm{m}$ cross-section waveguides support the fundamental TM guided mode, well below the cut-off around $1.6 \mu \mathrm{m}$ width, thus ensuring a single mode operation. The TE excitation is discarded by the TM polarized laser source, as well as the vertical invariance preventing undesired mode conversion.

\subsection{Maxwell equation, boundary conditions and power transmission}

In the considered Mid-IR bandwidth, SiGe and SiN are both linear, isotropic and non-dispersive dielectric materials with respective optical indices $n_{\mathrm{SiGe}}$ and $n_{\mathrm{SiN}}$. Since all sources are time-harmonic with pulsation $\omega$, the complete set of Maxwell equations reduces to two decoupled wave equations, respectively for the electric field $\mathbf{E}$ and magnetic field $\mathbf{H}$. In fact, the solution of only one wave equation, for instance 


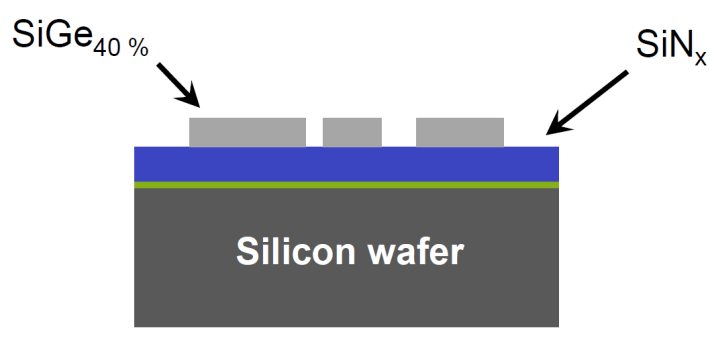

(a)

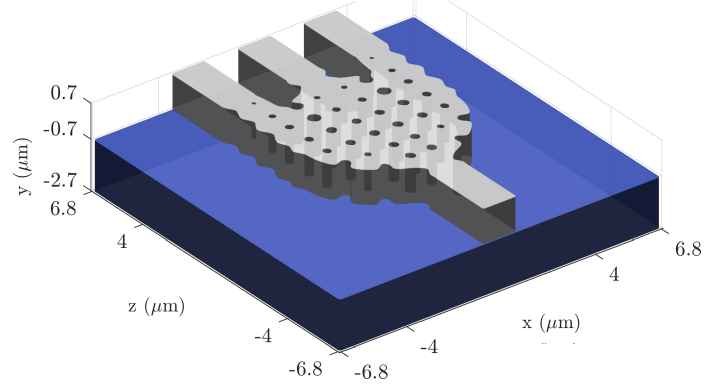

(b)

Fig. 1: The mid-IR platform is composed of $1.4 \mu \mathrm{m}$ thick SiGe waveguides (grey) etched on a $2 \mu \mathrm{m}$ thick silicon nitride substrate (blue). Dimensions are in $\mu \mathrm{m}$.

that for the electric field

$$
\nabla \times \nabla \times \mathbf{E}-k^{2} n^{2} \mathbf{E}=0 \text { with } k=\omega / c
$$

is required and the magnetic field is derived afterwards from the Maxwell-Faraday relation $\mathbf{H}=(\nabla \times$ $\mathbf{E}) /\left(i \omega \mu_{0}\right)$. Two types of boundary conditions are also applied in our problems.

The first one, namely "port" boundary condition on the waveguide entrance face $\Gamma_{\mathrm{in}}$, accounts for the injection of a given optical mode inside the nanophotonic components (Fig. 2). More precisely, in order to inject the $n^{\text {th }}$ guided mode this boundary condition uses the decomposition of the electric field in the waveguide as a sum of the $N$ incident and reflected guided modes ${ }^{1}$

$$
\mathbf{E}=\sum_{i=1}^{N} \alpha_{i} \underbrace{\mathbf{E}_{\text {mode }, i}}_{\text {incident }}+\beta_{i} \underbrace{\mathbf{E}_{\text {mode },-i}}_{\text {reflective }}
$$

as well as the orthogonality relation

$$
\int_{\Gamma_{\text {in }}}\left[\mathbf{E}_{\text {mode }, i} \times \mathbf{H}_{\text {mode }, j}^{*}\right] \cdot \mathbf{n} \mathrm{d} \mathbf{x}=0 \text { for }|i| \neq|j|
$$

to impose $\alpha_{i}=0$ for $i \neq n, \alpha_{n}=1$ (considering an injection power of $1 \mathrm{~W}$ ) while leaving free the $\beta_{i}$ reflection coefficients (see [11, Section 2.4.1] for full details).

The second type of boundary conditions is the "perfectly matched layer", defined on all the other faces of the computational domain, and allowing to simulate an infinite domain by adding areas that strongly absorb the incident waves while causing very few spurious reflections (see for instance $[9$, Section $9.6]$ or the original paper [4]).

The last important point to clarify concerns the objective of our optimization problem. Many nanophotonic components aim at maximizing power transmission from one waveguide to another, in other words at maximizing the flux of the Poynting vector on the output waveguide face $\Gamma_{\text {out }}$, defined by

$$
P=\frac{1}{2} \int_{\Gamma_{\text {out }}} \Re\left[\mathbf{E} \times \mathbf{H}^{*}\right] \cdot \mathbf{n} \mathrm{d} \mathbf{x},
$$

but it is also often desirable to control which modes propagate along the output waveguide. For this purpose, using decomposition (2) and relation (3) one can rewrite Equ. (4) as

$$
P=\sum_{i=1}^{N}\left|a_{i}\right|^{2}-\left|a_{-i}\right|^{2}
$$

with $a_{i}$ the following overlap integral between the electromagnetic field $(\mathbf{E}, \mathbf{H})$ and the $i^{\text {th }}$ mode $\left(\mathbf{E}_{\text {mode }, i}, \mathbf{H}_{\text {mode }, i}\right)$

$$
a_{i}=\frac{1}{4} \int_{\Gamma_{\text {out }}}\left[\mathbf{E} \times \mathbf{H}_{\text {mode }, i}^{*}+\mathbf{H} \times \mathbf{E}_{\text {mode }, i}^{*}\right] \cdot \mathbf{n} \mathrm{d} \mathbf{x} .
$$

1 We deliberately ignore here the treatment of the radiative modes for convenience, but their consideration does not change the results presented in this section. 


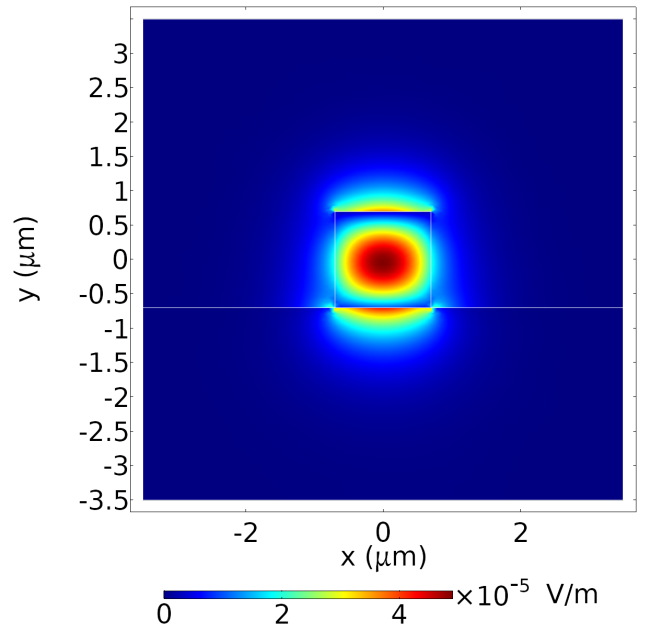

Fig. 2: $E_{y}$ component of the fundamental TM mode of a $\mathrm{Si}_{60} \% \mathrm{Ge}_{40} \% / \mathrm{SiNx}, 1.4 \mu \mathrm{m} \times 1.4 \mu \mathrm{m}$ wide waveguide at $\lambda=6 \mu \mathrm{m}$ (effective index $n_{\text {eff }}=2.5054$ ).

Thus, to maximize the power transported by the $m^{\text {th }}$ mode we only need to maximize $\left|a_{m}\right|^{2}$ instead of the total power $P$.

\section{Shape optimization}

Shape optimization is an inverse methodology that consists in determining the distribution of materials in a domain that fulfills a given objective. Mathematically speaking, we search for a shape $\Omega \subset \mathbb{R}^{3}$ (the repartition of SiGe inside the component) which maximizes a functional $J\left(\mathbf{E}_{\Omega}\right)$ (for instance the power transported by a given mode on the output waveguide) where $\mathbf{E}_{\Omega}$ corresponds to the solution of Maxwell equation (1) with the optical index $n(\mathbf{x})$ inside the component equal to $n_{\mathrm{SiGe}}$ (resp. $n_{\text {air }}$ ) if $\mathbf{x} \in \Omega$ (resp. $\mathbf{x} \notin \Omega)$.

Many approaches to such problems have been investigated in the literature [13] (the interested reader may also refer to a general review on shape and topology optimization algorithms [20]). The most popular one being the SIMP method [8] which relaxes the binary constraint on the optical index by allowing continuous variations of $n(\mathbf{x})$ inside the component between $n_{\text {SiGe }}$ and $n_{\text {air }}$, and uses a penalization of intermediate state or projections methods to finally obtain the distribution of the two materials. Evolutionary methods have also been considered several times [19,22] and have the advantage of producing inherently manufacturable design despite their high simulation cost. Geometrical methods [10,11] which, contrary to the SIMP methodology, directly deal with the binary-valued materials by iteratively advecting the shape, produce, after each iteration, a new shape better than the previous one in term of objective value. In the following section, we investigate a geometric shape optimization procedure based on Hadamard shape derivative [2, Chapter 6] and a level-set representation of the shape $[14,18]$.

\subsection{Hadamard shape derivative}

The goal of Hadamard shape derivative is to define the concept of "small variation" $\Omega+\delta \Omega$ of a shape $\Omega$ and thus to define the derivative of a functional $J$ according to a shape using a formula like

$$
" J^{\prime}(\Omega)=\lim _{\delta \Omega \rightarrow 0}(J(\Omega+\delta \Omega)-J(\Omega)) / \delta \Omega " .
$$

Once this derivative is determined and computed it allows us to derive a gradient-based algorithm.

The idea is that a shape $\Omega_{1}$ is a variation of $\Omega_{0}$ if $\Omega_{1}=(\operatorname{Id}+\boldsymbol{\theta})\left(\Omega_{0}\right)=\left\{\mathbf{x}+\boldsymbol{\theta}(\mathbf{x}), \mathbf{x} \in \Omega_{0}\right\}$ with $\boldsymbol{\theta}: \mathbb{R}^{3} \rightarrow \mathbb{R}^{3}$ a vector field. With this concept, $\Omega_{1}$ is said to be a small variation of $\Omega_{0}$ if $\boldsymbol{\theta}$ is small according to a given functional norm $\|\cdot\|$. In practice the considered norm is defined for smooth vector field $\boldsymbol{\theta}$ as $\|\boldsymbol{\theta}\|=\|\boldsymbol{\theta}\|_{\infty}+\|\nabla \boldsymbol{\theta}\|_{\infty}$ the sum of its maximal value and maximum local variation. 
We then define the shape derivative of $J$ at $\Omega$, named $J^{\prime}(\Omega)$, with the vector field given by the following expansion of $\boldsymbol{\theta} \mapsto J((\operatorname{Id}+\boldsymbol{\theta})(\Omega))$ at 0 :

$$
J((\operatorname{Id}+t \boldsymbol{\theta})(\Omega))=J(\Omega)+t\left\langle J^{\prime}(\Omega), \boldsymbol{\theta}\right\rangle+o(t)
$$

where $\langle\cdot, \cdot\rangle$ stands for the $L^{2}$ scalar product on $\partial \Omega$. In other words, if we take $\boldsymbol{\theta}=\varepsilon J^{\prime}(\Omega)$ with $\varepsilon>0$ small enough, the shape $(\operatorname{Id}+\boldsymbol{\theta})(\Omega)$ will be better than $\Omega$ since

$$
J((\operatorname{Id}+\boldsymbol{\theta})(\Omega)) \simeq J(\Omega)+\varepsilon\left\|J^{\prime}(\Omega)\right\|^{2}>J(\Omega) .
$$

The question is now how to compute $J^{\prime}(\Omega)$. For this, given the infinite number of vectors (i.e. dimensions) composing the vector field $J^{\prime}(\Omega)$, it is unthinkable to use finite differences. An adjoint-based method is then used and allows for the computation of the shape derivative at the only cost of an additional solution of a Maxwell-like equation ${ }^{2}$. This leads to the following formula of the shape derivative where A stand for the adjoint solution and $T$ for the thickness of the component

$$
\begin{aligned}
& J^{\prime}(\Omega)=k^{2} \int_{T} \Re {\left[\left(n_{\mathrm{SiGe}}^{2}-n_{\text {air }}^{2}\right)(\mathbf{n} \times \mathbf{E} \times \mathbf{n}) \cdot\left(\mathbf{n} \times \mathbf{A}^{*} \times \mathbf{n}\right)\right.} \\
&\left.-\left(n_{\mathrm{SiGe}}^{-2}-n_{\text {air }}^{-2}\right) \mathbf{n} \cdot\left(n^{2} \mathbf{E}\right) \cdot \mathbf{n} \cdot\left(n^{2} \mathbf{A}^{*}\right)\right] d y \mathbf{n}
\end{aligned}
$$

and where it is interesting to note that the tangential $(\mathbf{n} \times \mathbf{E} \times \mathbf{n})$ and normal $(\mathbf{n} \cdot \mathbf{E})$ components of the fields are treated differently since only the tangential part of $\mathbf{E}$ is continuous across the interface between materials. Note also that the integral along $T$ gives an $y$-invariant vector field $J^{\prime}(\Omega)$ and thus an $y$-invariant shape, as produced by the etching process).

\subsection{Level-set representation}

Numerically the shape is defined through a level-set function $\phi([18,1])$, that is to say $\partial \Omega=\{\mathbf{x}, \phi(\mathbf{x})=0\}$ and $\Omega=\{\mathbf{x}, \phi(\mathbf{x})<0\}$ (Fig. 3). This representation eases the numerical process since we have the relation $\mathbf{n}(\mathbf{x})=\nabla \phi(\mathbf{x}) /\|\nabla \phi(\mathbf{x})\|$ on $\partial \Omega$ and the fact that a level-set function $\phi_{\boldsymbol{\theta}}$ for $(\mathrm{Id}+\varepsilon \boldsymbol{\theta})(\Omega)$ may be obtained by solving the following equation

$$
\partial_{t} \psi(t, \mathbf{x})+\boldsymbol{\theta}(\mathbf{x}) \cdot \nabla_{\mathbf{x}} \psi(t, \mathbf{x})=0 \text { with } \psi(0, \mathbf{x})=\phi(\mathbf{x})
$$

and setting $\phi_{\boldsymbol{\theta}}(\mathbf{x})=\psi(\varepsilon, \mathbf{x})$. In practice $\boldsymbol{\theta}$ is always in the same direction as $\mathbf{n}$ on $\partial \Omega$. This fact reduces Equation (9) to a Hamilton-Jacobi equation for which there exist fast solvers [18, Section 6.4].

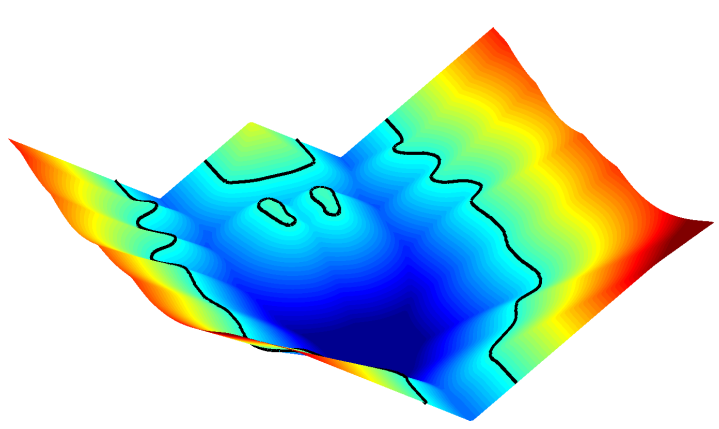

(a)

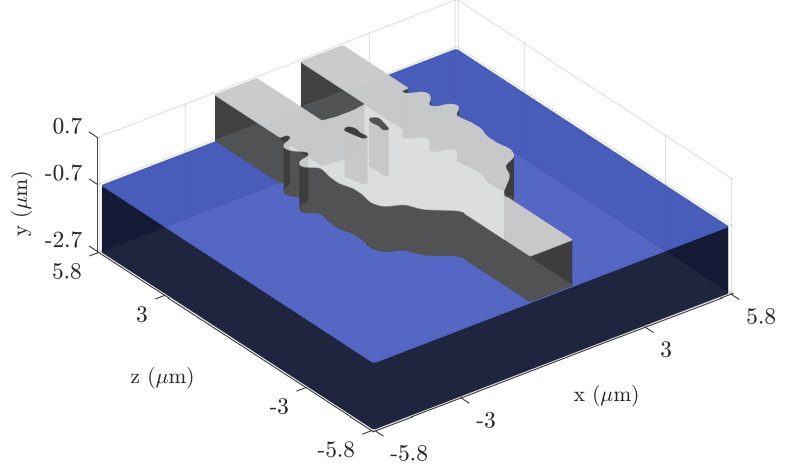

(b)

Fig. 3: Level-set representation of the shape $\Omega$ of Fig. 6(a) with 0 level-set $(\partial \Omega)$ in black.

\footnotetext{
2 In a nutshell this adjoint equation corresponds to a Maxwell equation simulation, where the mode is injected from the output waveguide with a power proportional to the value of $a_{n}^{*}$ (the propagating mode power considered in the objective $J(\Omega)$ ). For more details see [11].
} 


\subsection{Manufacturability and robustness}

The previous subsection provides a general algorithm to optimize and therefore get the optimal design of many nanophotonic components. The problem then comes from the fact that most of the shapes thus obtained will not be manufacturable (see Section 4), and, even if manufactured could be too sensitive (not robust) to the realization process to be usable.

To solve the first problem, a method close to the one proposed in [16] is used. In this paper the authors propose to periodically advect the shape along the curvature of the shape, that is to say consider the vector field $\boldsymbol{\theta}=\kappa \mathbf{n}$ (with $\kappa(\mathbf{x})$ the local curvature on $\Omega$ at $\mathbf{x}$ ) and solve Equation (9) for a small time step in order to flatten the shape. It was not pointed out in this article, but it is interesting to notice, that this methodology is equivalent to considering a penalization of the perimeter on the objective since we have the following expansion:

$$
\operatorname{Per}((\operatorname{Id}+\boldsymbol{\theta})(\Omega))=\operatorname{Per}(\Omega)+\int_{\partial \Omega} \boldsymbol{\theta} \cdot \mathbf{n} \kappa d s+o(\boldsymbol{\theta}) \text { with } \operatorname{Per}(\Omega)=\int_{\partial \Omega} d s .
$$

The penalization coefficient in front of the perimeter acts as the time step in Equation (9).

Concerning the robustness issue we use our previously proposed methodology [11] considering that the manufacturing process will lead to a general homogeneous erosion or dilation of the shape (which means that for $\Omega$ the resulting shape will be $(\operatorname{Id}+\delta \mathbf{n})(\Omega)=\Omega_{\delta}$ with $\delta$ a bounded unknown parameter $)$. Briefly, the worst-case objective is discretized as:

$$
\min _{|\delta|<C} J((\operatorname{Id}+\delta \mathbf{n})(\Omega))=J\left(\Omega_{\delta}\right) \approx \min _{\delta \in\left\{\delta_{1}, \ldots, \delta_{n}\right\}} J\left(\Omega_{\delta}\right) .
$$

Computing each $\Omega_{\delta_{i}}$ (using the $n$ simulations of $\mathbf{E}_{\Omega_{\delta_{i}}}$ and their associated adjoint) we know how to modify each $\Omega_{\delta_{i}}$ (not $\Omega$ ) in order to optimize $J\left(\Omega_{\delta_{i}}\right)$. We then prove the following shape derivative for $J_{\delta}(\Omega)=J\left(\Omega_{\delta}\right)$ :

$$
J_{\delta}^{\prime}(\Omega)=J^{\prime}\left(\Omega_{\delta}\right) \circ(\operatorname{Id}+\delta \mathbf{n})|\operatorname{det}(\operatorname{Id}+\delta \nabla \mathbf{n})|\left((\operatorname{Id}+\delta \nabla \mathbf{n})^{-1} \mathbf{n}\right) \cdot \mathbf{n}
$$

which allows to optimize $J\left(\Omega_{\delta_{i}}\right)$ by only modifying $\Omega$. Finally to optimize simulatenously the $n$ objectives $J\left(\Omega_{\delta_{i}}\right)$, a multi-gradient descent algorithm is used.

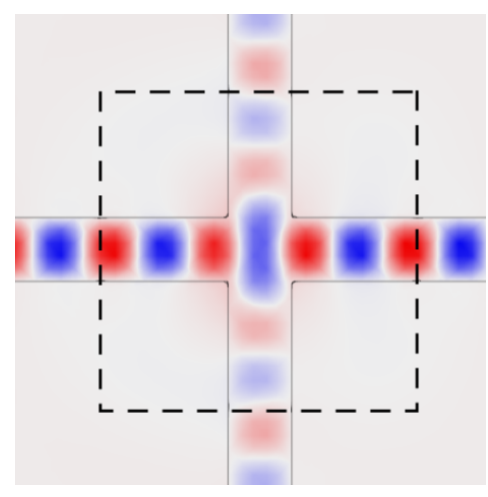

(a) Computation of the field distribution inside the component. Field leakage in the perpendicular waveguides is observed.

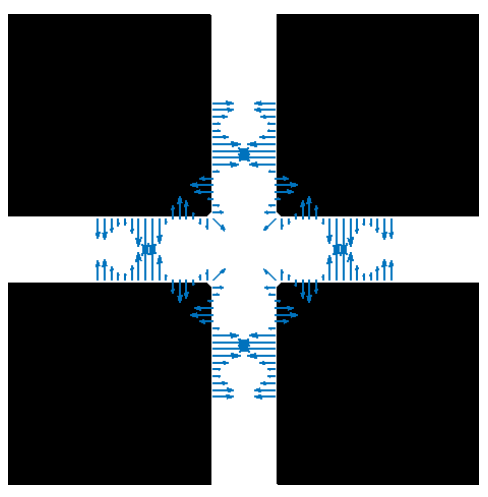

(b) The shape gradient field is computed and gives informations on how to modify the borders of the shape $\Omega$.

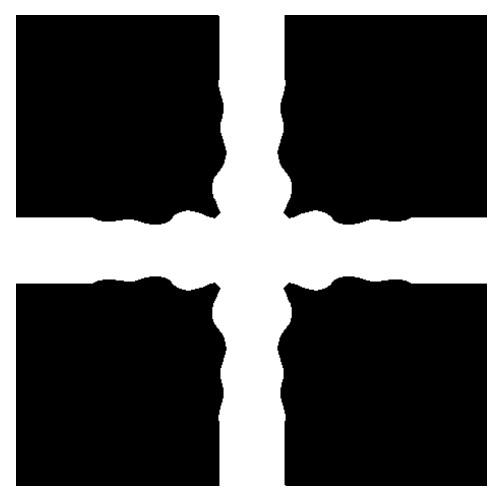

(c) The material distribution is modified according to the shape gradient.

Fig. 4: The three main steps of one iteration of the geometrical shape optimization algorithm used in this paper to optimize a nanophotonic waveguides crossing. The fundamental mode is injected in the left waveguide.

The final optimization algorithm is sketched in Fig. 4. Let us note that we have added a simple linear search method to accelerate the convergence of the optimization process and minimize problems related 
to numerical oscillations in the gradient descent algorithm. At each iteration, if the objective does not decrease by more than $2 \%$ compared to the previous iteration then the modification of the shape is accepted and the descent step is increased for the next iteration (multiplied by 1.1). Otherwise, the shape is not modified and the current iteration is restarted with a descent step divided by two.

The three-dimensional solution of the direct (1) and the adjoint problems are performed by means of the finite element method, as implemented in the COMSOL Multiphysics commercial software (COMSOL AB, Stockholm, Sweden). The global algorithm, as well as the advection of the level-set are implemented in MATLAB (The MathWorks Inc., Natick, U.S.A.).

\section{Results}

The methodology presented above has firstly been applied to several test cases in the context of the mid-IR platform. Then, in following sub-sections, the issues of fabricability and robustness are tackled.

\subsection{Example components}

The first test case consists in a compact waveguide crossing (Fig. 5): light is injected in the first TM mode in the left guide and the objective is to maximize the transmission in the same mode in the right guide, through the junction. The shape evolves in the $8 \mu \mathrm{m} \times 8 \mu \mathrm{m}$ dashed square, from initial conditions to a converged one after less than 400 iterations (Fig. 5(a)). It is not surprising that the optimum reached by the algorithm is sensitive to the chosen initial conditions (insets in Fig. 5(b) and (c)). However, in both cases, around $92 \%$ of the input power is transmitted through the crossing. Let us note that some of the void features that are present in the device of Fig. 5(b) are smaller than the expected resolution of the manufacturing process and thus would not be correctly reproduced in the actual component. The issue of fabricability is discussed in section 4.2 .

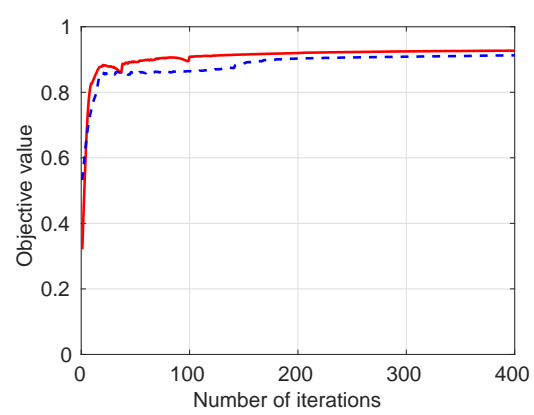

(a)

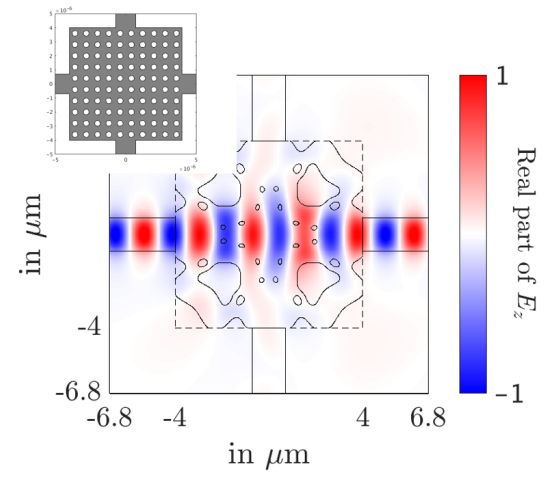

(b)

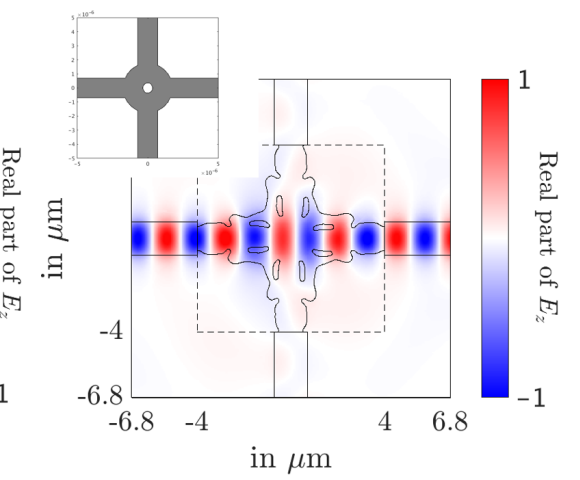

(c)

Fig. 5: Convergence plot (a) of two waveguide crossings obtained with different initial conditions (b) and (c). Initial shapes are in insets. The normal component of the electric field is plotted.

In the power splitters test cases (Fig. 6), light is injected in the first TM mode in the lower guide. Equally shared power transmission is expected in each of the top waveguides. Due to the components symmetry, a single objective is sought in the two channels case. However, in the three channels case two objectives are aimed at, and the multi-gradient descent algorithm is harnessed in order to improve both objectives simultaneously. The shape evolves from different initial conditions to a converged one after a few hundreds iterations. In the $6 \mu \mathrm{m} \times 6 \mu \mathrm{m}$ two channels power splitter, $49 \%$ of the power is transmitted in each exit guide and in the $8 \mu \mathrm{m} \times 8 \mu \mathrm{m}$ three channels power splitter, $32-33 \%$ of the power is transmitted in each exit guide. These performances can be considered as satisfactory, relative to the small footprint of the devices. Let us note that, at this wavelength and on a similar mid-IR platform, conventional components fulfilling the power splitting function, such as multimode interference couplers 
or directional couplers would have much better insertion losses, but respectively span over more than $60 \mu \mathrm{m}$ or $300 \mu \mathrm{m}$.

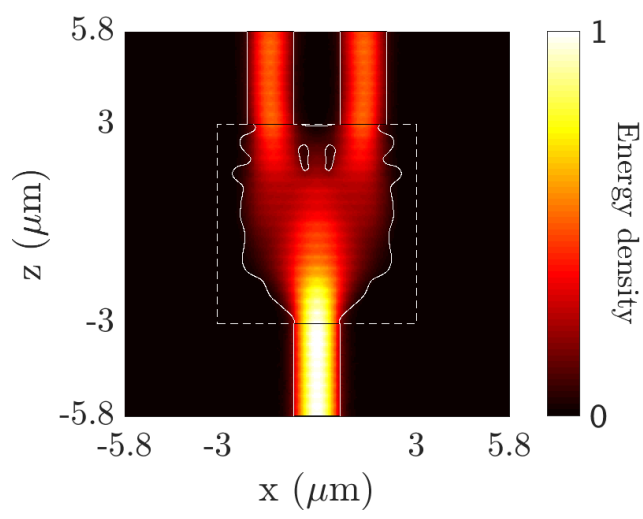

(a)

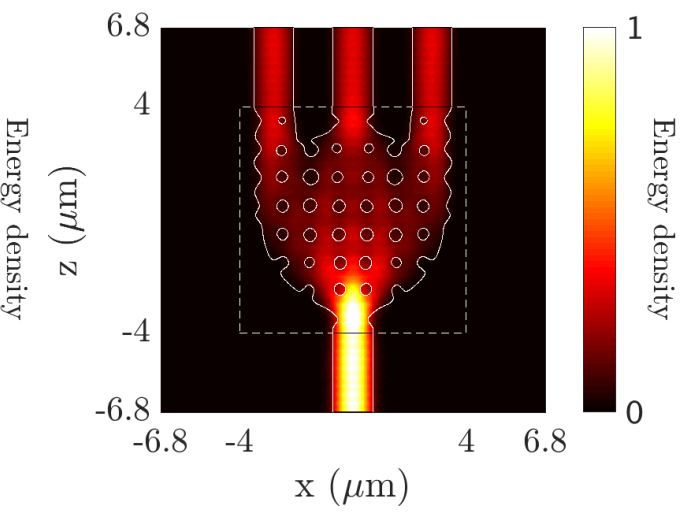

(b)

Fig. 6: Two and three channels power splitters. The power splitting ratio is $49 \%-49 \%$ in (a) and $32 \%-33 \%-32 \%$ in (b). The energy density is plotted.

The duplexer test case (Fig. 7) constitutes another multiobjective optimization problem. Here, in order to minimize the cross-talk, the objectives are defined, at each wavelength, as the difference between the output power at the two exit waveguides. Transmission in the exit waveguide reaches $-1.8 \mathrm{~dB}$ at $5.9 \mathrm{\mu m}$ and $-1.7 \mathrm{~dB}$ at $6.2 \mu \mathrm{m}$, while cross-talk is limited to respectively $-15 \mathrm{~dB}$ and $-13 \mathrm{~dB}$.

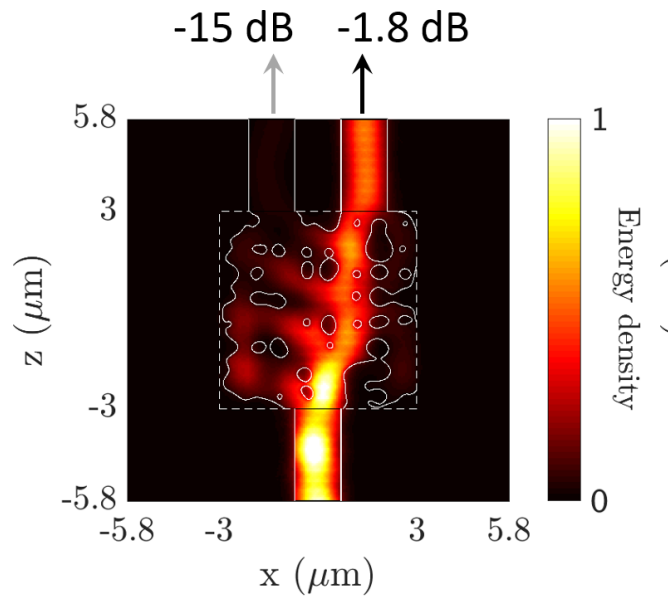

$5.9 \mu \mathrm{m}$

(a)

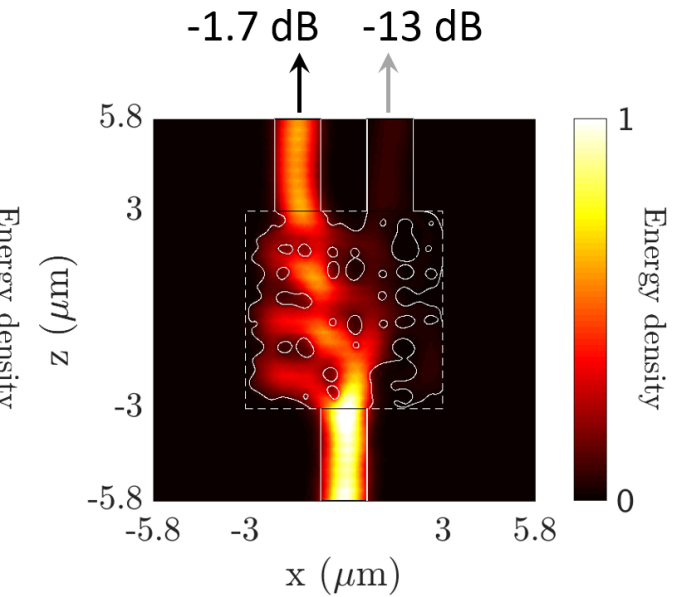

$6.2 \mu \mathrm{m}$

(b)

Fig. 7: Wavelength duplexer at $5.9 \mu \mathrm{m}$ and $6.2 \mu \mathrm{m}$. The optimization is performed in order to limit the cross-talk between the output waveguides. The energy density is plotted.

\subsection{Fabricability}

As previously observed on Fig. 5 to 7 , the algorithm can produce tiny voids, that are smaller than the expected resolution of the fabrication process. Regaining control on the size of the void or island features is an important issue for the upcoming deployment in real applications. The problem can be solved, for instance, by eliminating them with an image processing step, either during the optimization process or on the final output [16]. Here instead, a penalization term, depending on the shape perimeter, is added to 
the objective function. The effect is to decrease the average curvature of the shape, and thus to eliminate the smallest features.

An illustration is presented on the case of a three channels power splitter (Fig. 8). As can be seen on Fig. 8(a), where a disk of $300 \mathrm{~nm}$ is drawn, the output of the initial optimization algorithm includes a few non fabricable voids, for instance in the vicinity of the output waveguides. At the expense of a relatively low performance loss, these small features are eliminated by the penalized algorithm (Fig. 8(b)). However, if the value of the penalization coefficient is further increased, excessively smooth designs are produced, that are too lossy to be considered (Fig. 8(c)).

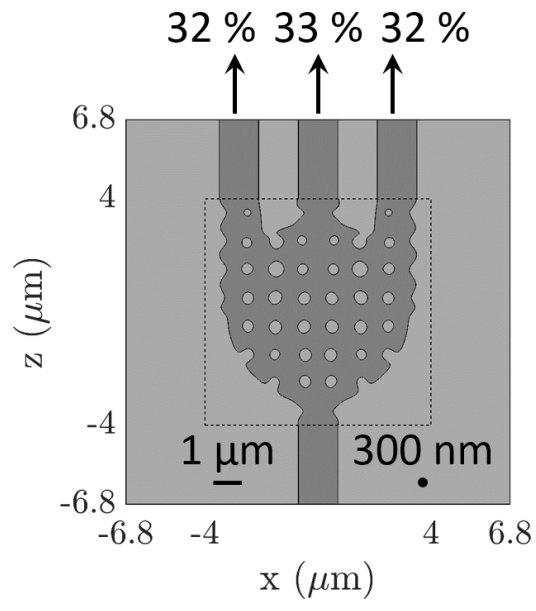

(a)

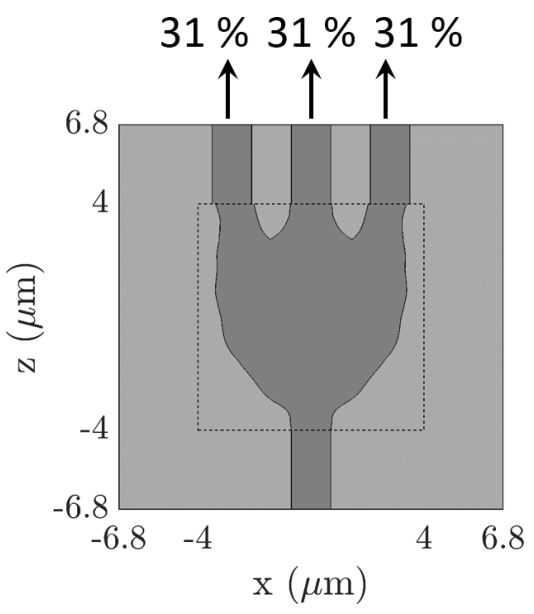

(b)

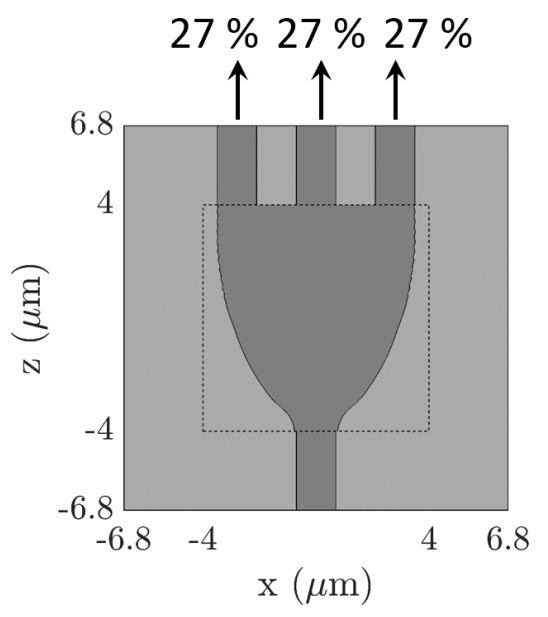

(c)

Fig. 8: Power splitter. Initial design (a) and two fabricable designs (b) and (c). The expected process resolution of $300 \mathrm{~nm}$ is depicted by the black circle in subplot (a).

\subsection{Bandwidth}

One of the key characteristics to evaluate the performance of the components is the optical transmission spectrum. It is presented on Fig. 9 for the components previously designed. An algorithm aiming at the improvement of the bandwidth has been presented in a previous paper [11].

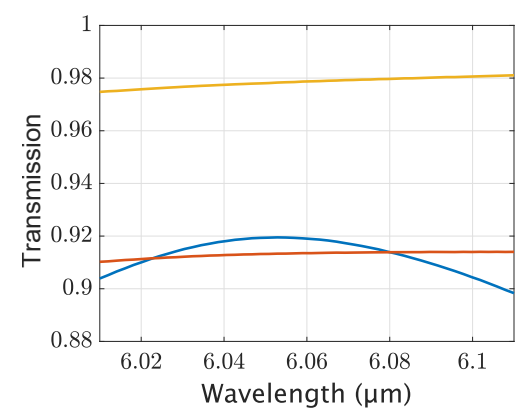

(a)

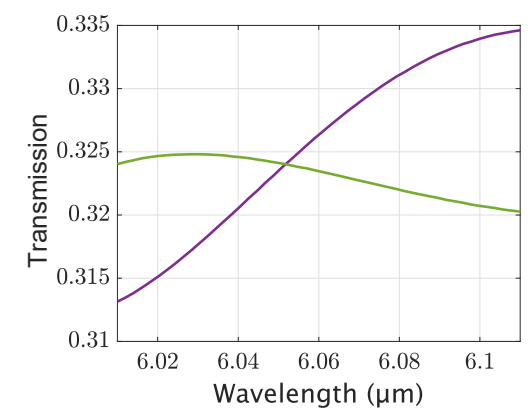

(b)

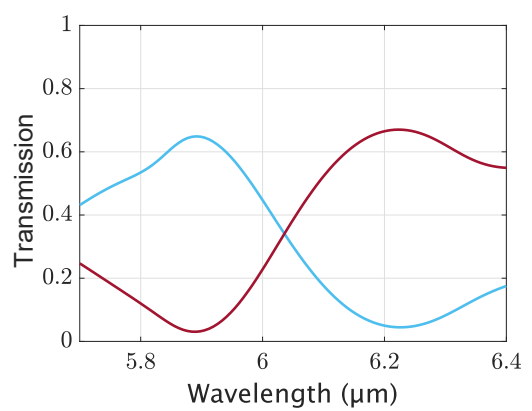

(c)

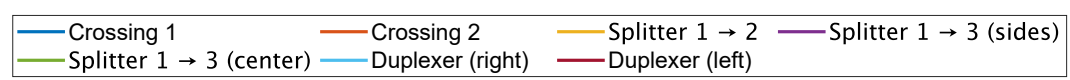

Fig. 9: Wavelength robustness of previously considered devices. Wavelength transmission spectrum of the crossings and the two channels power splitter (a), of the three channels power splitter (b) and of the duplexer (c). 


\subsection{Robustness}

Due to uncertainties in the lithography and etching micro-fabrication steps, the components actually manufactured slightly differ from the ideal shape provided by optimization. The study of the sensitivity of the designs obtained previously (see Fig. 10) with respect to over- or under-etching, show that those with few small patterns are not very responsive to manufacturing uncertainties while those composed of more complex topologies, such as the duplexer, have a much less robust behavior. Thus, producing robust designs, i.e. designs that are as insensitive as possible to fabrication flaws, is highly desirable in many practical cases, such as the one of the mirror considered below.

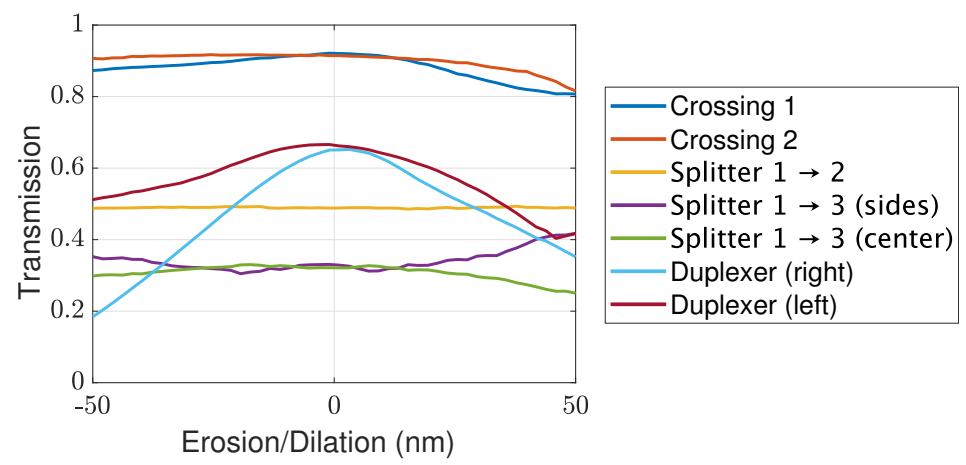

Fig. 10: Robustness to under and over etching of previously considered devices.

The method used here consists in (i) modeling lithography and etching globally, by an homogeneous erosion or dilation of the shape, (ii) defining several objectives, respectively on the reference, eroded and dilated shapes, and (iii) carrying out a worst case robustness scenario relying on the previously used multi-gradient descent optimization algorithm.

A compact mirror is used as a test case to demonstrate the efficiency of the robust design algorithm. The component design obtained after 400 iterations of the reference optimization algorithm (Fig. 11(a)) has a reflectivity $\approx 96 \%$. It is used as an initial condition for the robust design process. During the first iterations, as can be seen on the convergence curve presented on Fig. 11(b), the reflectivity of the $\pm 50 \mathrm{~nm}$ eroded and dilated shapes are significantly lower than that of the nominal shape. However, after a few dozen iterations, all three objectives gather together and continue their progression towards an enhanced final solution, robust to fabrication uncertainties (Fig. 11(c)).

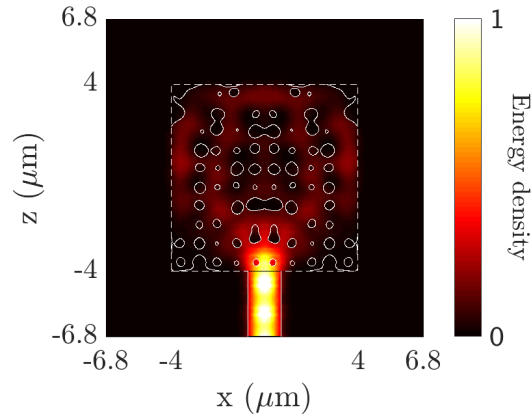

(a)

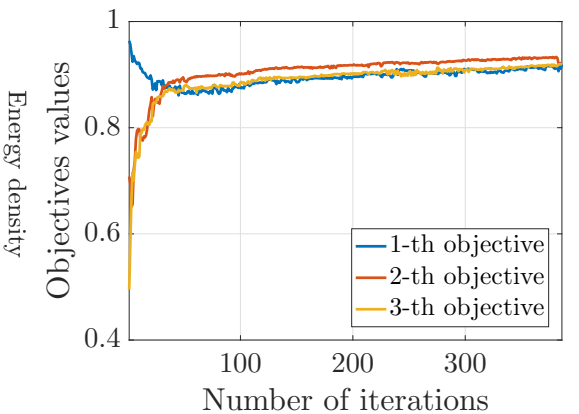

(b)

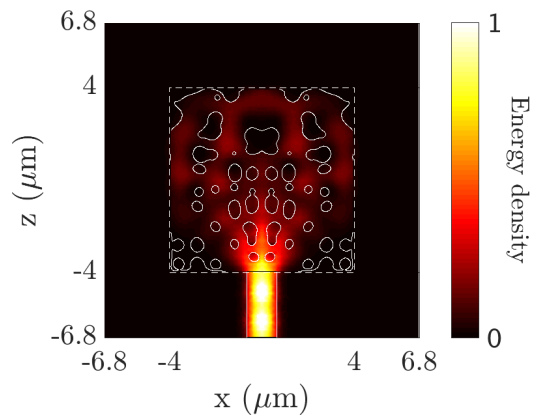

(c)

Fig. 11: Robust design algorithm. Non robust mirror used for initialization (a), convergence plot of the robust design algorithm (b) and design robust to $\pm 50 \mathrm{~nm}$ fabrication uncertainty (c). The energy density is plotted. 
The reflectivity of several mirrors is plotted against the distance of erosion or dilation on Fig. 12(a). The reflectivity of the non robust design (black curve) is dramatically lowered as soon as the erosion or dilation exceeds $\pm 50 \mathrm{~nm}$. On the contrary, at the expense of a slight degradation of the maximum performance, the $\pm 25 \mathrm{~nm}$ (red curve) and $\pm 50 \mathrm{~nm}$ (green curve) robust designs retain a flat response on their respective variation interval. The loss is more important for the $\pm 100 \mathrm{~nm}$ (blue curve) robust design, which has been obtained by optimizing simultaneously on five objectives consisting on equally spaced eroded and dilated shapes. Let us note however that a $\pm 100 \mathrm{~nm}$ process uncertainty is excessively pessimistic. Let us note that the correlation between geometrical robustness and wavelength robustness, which has already been noticed previously [15] is confirmed by our computations. As can be seen on Fig. 12(b), the response of the $\pm 25 \mathrm{~nm}$ and $\pm 50 \mathrm{~nm}$ robust designs are flater than the one of the initial mirror.

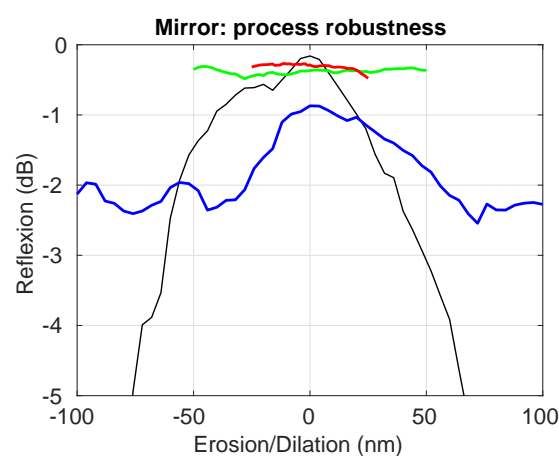

(a)

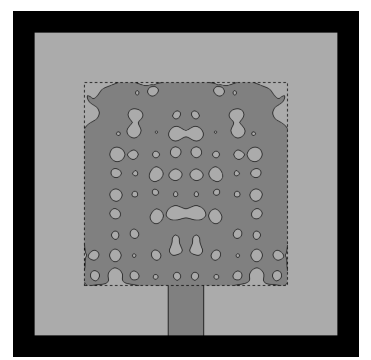

(c) Initial design $\mathrm{R}_{\text {nominal }} \approx 96 \%$

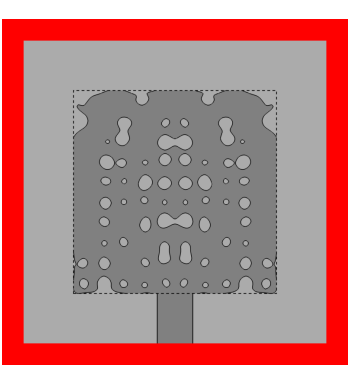

(d) Robust $\pm 25 \mathrm{~nm}$ $\mathrm{R}_{\text {nominal }} \approx 94 \%$

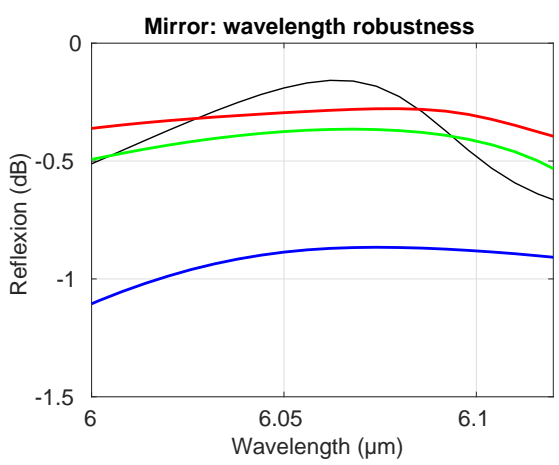

(b)

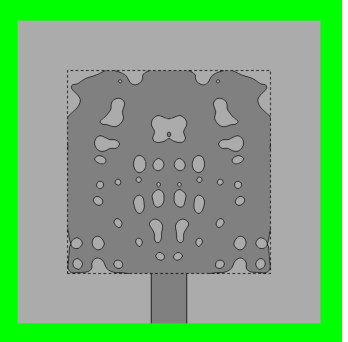

(e) Robust $\pm 50 \mathrm{~nm}$ $\mathrm{R}_{\text {nominal }} \approx 92 \%$

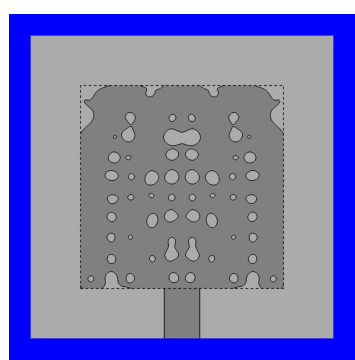

(f) Robust $\pm 100 \mathrm{~nm}$ $\mathrm{R}_{\text {nominal }} \approx 82 \%$

Fig. 12: Robustness to fabrication uncertainty at $6.06 \mu \mathrm{m}(\mathrm{a})$, device bandwidth around $6.06 \mu \mathrm{m}$ (b) and the initial and robust nominal designs (c)-(f). A $100 \%$ (resp. $50 \%$ ) reflectivity corresponds to 0 $\mathrm{dB}$ (resp. $-3 \mathrm{~dB}$ ). The black curve represents the non robust design and the red, green and blue curves respectively represent the $\pm 25 \mathrm{~nm}, \pm 50 \mathrm{~nm}$ and $\pm 100 \mathrm{~nm}$ robust designs.

\section{Conclusion}

In this paper, our shape derivative based methodology, dedicated to the optimization of photonic components has been summarized and several components designed for the mid-IR have been presented. To the best of our knowledge, this constitutes the first attempt to address this wavelength range. The issues of fabricability and robustness to manufacturing uncertainties have been tackled.

When compared to conventional components, the very small footprint of the shape optimized building blocks is hindered by degraded performances in terms of insertion losses. Thus, additional progress is needed to fully take advantage of their high density integration potential and find a way towards actual applications in photonic integrated circuits [13].

In the near future, a more comprehensive library of mid-IR passive components will be developed and the fabrication and tests in the CEA-LETI facilities will be undertaken. 
Acknowledgements This research was partly funded by the European Union project REDFINCH (E.U. H2020, ${ }^{\circ}$ 780240). Moreover, the authors are grateful to J.-M. Fedeli for his expertise regarding the technological platform and for his involvement in the future fabrication of the micro-components.

\section{References}

1. Allaire, G., Jouve, F., Toader, A.M.: Structural optimization using sensitivity analysis and a level-set method. Journal of computational physics 194(1), 363-393 (2004)

2. Allaire, G., Schoenauer, M.: Conception optimale de structures, vol. 58. Springer (2007)

3. Bendsøe, M.P., Sigmund, O.: Topology optimization: theory, methods, and applications. Springer, Berlin; New York (2003)

4. Berenger, J.P.: A perfectly matched layer for the absorption of electromagnetic waves. Journal of computational physics 114(2), 185-200 (1994)

5. Brun, M., Labeye, P., Grand, G., Hartmann, J.M., Boulila, F., Carras, M., Nicoletti, S.: Low loss SiGe graded index waveguides for mid-IR applications. Optics Express 22(1), 508-518 (2014).

6. Fedeli, J., Nicoletti, S.: Mid-Infrared (Mid-IR) Silicon-Based Photonics. Proceedings of the IEEE pp. 1-11 (2018)

7. Frellsen, L.F., Ding, Y., Sigmund, O., Frandsen, L.H.: Topology optimized mode multiplexing in silicon-on-insulator photonic wire waveguides. Optics Express 24(15), 16866-16873 (2016)

8. Jensen, J.S., Sigmund, O.: Topology optimization of photonic crystal structures: a high-bandwidth low-loss t-junction waveguide. JOSA B 22(6), 1191-1198 (2005)

9. Jin, J.M.: The finite element method in electromagnetics. John Wiley \& Sons (2015)

10. Lalau-Keraly, C.M., Bhargava, S., Miller, O.D., Yablonovitch, E.: Adjoint shape optimization applied to electromagnetic design. Optics express 21(18), 21693-21701 (2013)

11. Lebbe, N., Dapogny, C., Oudet, E., Hassan, K., Gliere, A.: Robust shape and topology optimization of nanophotonic devices using the level set method. HAL preprint hal-01860882 (2018). URL https://hal.archives-ouvertes.fr/hal01860882

12. Lu, J., Vučković, J.: Nanophotonic computational design. Optics Express 21(11), 13351 (2013)

13. Molesky, S., Lin, Z., Piggott, A.Y., Jin, W., Vučković, J., Rodriguez, A.W.: Outlook for inverse design in nanophotonics. arXiv preprint arXiv:1801.06715 (2018). URL https://arxiv.org/abs/1801.06715

14. Osher, S., Fedkiw, R.: Level set methods and dynamic implicit surfaces, vol. 153. Springer Science \& Business Media (2006)

15. Piggott, A.Y., Lu, J., Lagoudakis, K.G., Petykiewicz, J., Babinec, T.M., Vučković, J.: Inverse design and demonstration of a compact and broadband on-chip wavelength demultiplexer. Nature Photonics 9(6), 374-377 (2015)

16. Piggott, A.Y., Petykiewicz, J., Su, L., Vučković, J.: Fabrication-constrained nanophotonic inverse design. Scientific Reports 7(1), 1786 (2017)

17. Pironneau, O.: Optimal shape design for elliptic systems. In: System Modeling and Optimization, pp. 42-66. Springer (1982)

18. Sethian, J.A.: Level set methods and fast marching methods: evolving interfaces in computational geometry, fluid mechanics, computer vision, and materials science, vol. 3. Cambridge university press (1999)

19. Shen, B., Wang, P., Polson, R., Menon, R.: An integrated-nanophotonics polarization beamsplitter with $2.4 \times 2.4 \mu \mathrm{m}$ 2 footprint. Nature Photonics 9(6), 378 (2015)

20. Sigmund, O., Maute, K.: Topology optimization approaches. Structural and Multidisciplinary Optimization 48(6), 1031-1055 (2013)

21. Soref, R.: Mid-infrared photonics in silicon and germanium. Nature photonics 4(8), 495 (2010)

22. Yu, Z., Cui, H., Sun, X.: Genetic-algorithm-optimized wideband on-chip polarization rotator with an ultrasmall footprint. Optics letters 42(16), 3093-3096 (2017)

23. Zhou, W., Cheng, Z., Wu, X., Sun, X., Tsang, H.K.: Fully suspended slot waveguide platform. Journal of Applied Physics 123(6), 063103 (2018). 